\title{
CAMBRJDGE
}

\section{Observation Medicine}

\section{Principles and Protocols}

Sharon Mace, Department of Emergency Medicine, Cleveland Clinic, Ohio April 2017 / 9781107022348 / Hardback / \$105.00 / f64.99

Using sample administrative and clinical protocols that any hospital can use, this book gives a detailed account of how to set up and run an observation unit and reviews all medical conditions in which observation medicine may be beneficial. In addition to clinical topics such as improving patient outcomes and avoiding readmissions, it also includes practical topics such as design, staffing, and daily operations; fiscal aspects, such as coding, billing, and reimbursement; regulatory concerns, such as aligning case management and utilization review with observation; nursing considerations; and more. The future of observation medicine, and how it can help solve the healthcare crisis from costs to access, is also discussed. Although based on US practices, this book is also applicable to an international audience, and contains instructions for implementing observation in any setting or locale and in any type of hospital or other appropriate facility.

- Contains clinical protocols for diseases/conditions that may be managed by observation medicine, which is an up-and-coming solution to the crisis of costs and access to emergency healthcare

- Contains administrative protocols and covers the financial and business aspects of running an observation unit

- Offers an international perspective, with practical methods for implementing observation medicine in any location and with any type of resources, staffing, and education
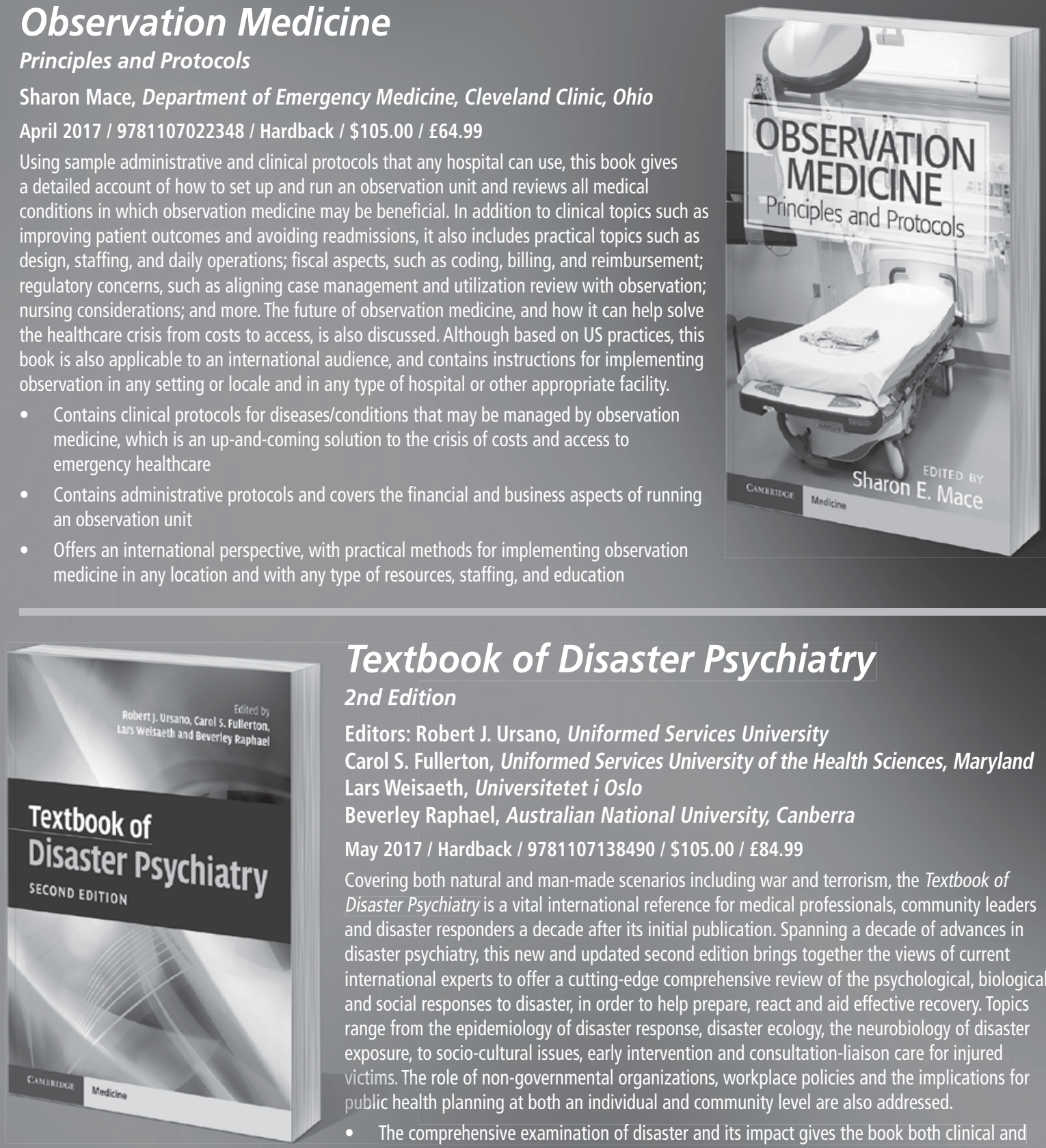

\section{Textbook of Disaster Psychiatry}

\section{2nd Edition}

Editors: Robert J. Ursano, Uniformed Services University

Carol S. Fullerton, Uniformed Services University of the Health Sciences, Maryland Lars Weisaeth, Universitetet i Oslo

Beverley Raphael, Australian National University, Canberra

May 2017 / Hardback / 9781107138490 / \$105.00 / £84.99

Covering both natural and man-made scenarios including war and terrorism, the Textbook of Disaster Psychiatry is a vital international reference for medical professionals, community leaders and disaster responders a decade after its initial publication. Spanning a decade of advances in disaster psychiatry, this new and updated second edition brings together the views of current international experts to offer a cutting-edge comprehensive review of the psychological, biological and social responses to disaster, in order to help prepare, react and aid effective recovery. Topics range from the epidemiology of disaster response, disaster ecology, the neurobiology of disaster exposure, to socio-cultural issues, early intervention and consultation-liaison care for injured victims. The role of non-governmental organizations, workplace policies and the implications for public health planning at both an individual and community level are also addressed.

- The comprehensive examination of disaster and its impact gives the book both clinical and public health relevance

- The text has been updated to cover a decade of advances, leading to more effective approaches to preparation, treatment, intervention and therefore recovery

- Concepts and approaches are applied to worldwide needs, resulting in a text relevant to real life practice

\section{For more information visit:} www.cambridge.org 


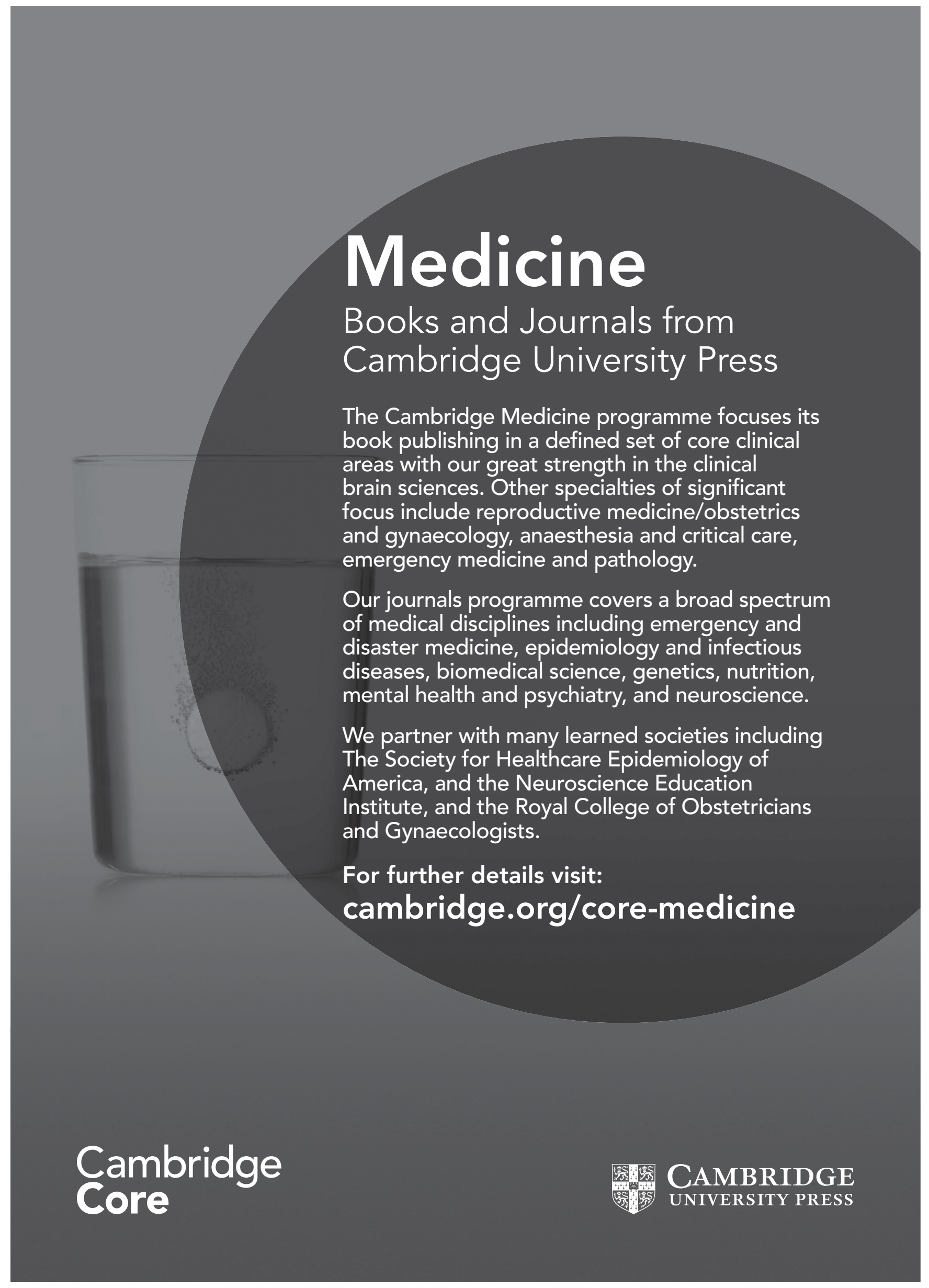

